\title{
Chained Displays: Configuration of Multiple Co-Located Public Displays
}

\author{
Amir E. Sarabadani Tafreshi, Milan Bombsch and Gerhard Tröster \\ ETH Zürich, CH-8092 Zürich, Switzerland
}

\begin{abstract}
Networks of pervasive display systems involving public and semi-public displays have allowed experiences to be created that span across multiple displays to achieve a stronger effect on the viewers. However, little research has been done so far on the configuration of content for multiple displays, especially when encountered in sequence in what is commonly referred to as chained displays. As a first step towards determining appropriate configuration strategies for chained displays, we have identified and investigated different approaches for configuring content. We report on a user study on the effect of the different configuration models in terms of usability and user engagement.
\end{abstract}

\section{KEYWORDS}

Configuration model; Multiple displays; Pervasive display; Content; usability; user engagement.

\section{Introduction}

Large displays have become so popular in the workplace and in public areas that pervasive display systems (PDS) are now in everyday use. The content shown on such displays vary; some involve educational, news, advertising, and/or events content. Much recent research on Pervasive Display Systems has focused on global networks of displays and associated sensors to potentially form a novel communication medium [1]. This not only allows developers to create new and innovative applications, but also introduces the concept of chained displays which are sets of displays encountered in sequence. Chained displays could coordinate their content in such a way that it offers viewers a new and potentially more influential experience. However, which content configuration strategy would be appropriate for chained displays is still an open question.

To address this gap, our goal is to explore different configuration strategies and evaluate their potential effect on viewers. As a first step, we set out to identify and investigate alternative configuration models. We first used workshop-style brainstorming sessions, together with a study of the research literature and reported configurations of current chained display systems, to identify a set of configuration models covering a range of scenarios and use cases. We provide a detailed overview of these models and their features, and report on a user study concerning usability and user engagement. We show that the most commonly used configuration for chained displays is suboptimal and improvements can be made by using other configurations. 
We implemented support for chained displays and our configuration models in a PDS platform called ScreenPress [2] which was developed previously for the rapid prototyping of public display network applications. We show how such a system can support chained display configurations.

We begin with a discussion of related work and then present the set of configuration models that we have identified. After detailing our implementation of the configuration models, we describe two prototypes of chained configurations. We then report on our user study on the usability and user engagement effects of these configuration models. The paper concludes with a discussion on the results of the study, followed by final remarks outlining the limitations and future research directions.

\section{Related Work}

Unlike personal devices [3], public displays are known to frequently go unnoticed [1]. Therefore, the issues of how to attract and engage viewers are well-known problems in PDS [4]. Multiple previous studies have explored the display blindness and various ways to address the blindness issue, e.g. by displaying a mirrored user shadow or silhouette to attract the viewers' attention [5] or adapting the content to improve the perception of viewers [6-8].

Previous research has investigated the many different factors that can have an impact on viewer engagement. For example, [9] found that the distance between the viewer and the display can affect the engagement of the viewers, while [4] showed that relevant content that meets viewer interests can increase their level of attention. Another factor concerns the size and number of the displays [10]. The location of a PDS is also considered as one of the essential factors, with lighting and contrast conditions as well as other location-based factors influencing how well people are able to view and interact with the displays $[9,11]$.

However, relatively little research has addressed the potential to improve viewer engagement by coordinating content across multiple displays. In particular, the idea of chained displays which configure and coordinate content across a set of displays encountered by a viewer in sequence has been put forward as a possible way of increasing user engagement, but has still to be fully explored.

One area of research on chained displays has been to investigate the physical configuration in terms of shapes and positions. For example, [12] examined the effect of different position configurations of chained displays, e.g. flat, circular or hexagonal. They found that a flat configuration had the strongest effect of all the tested configurations. Meanwhile other researchers have investigated the shape-changing possibilities of chained displays [13-15].

In terms of strategies used to coordinate content displayed on multiple displays, the scenarios previously envisaged in the literature or used in traditional setups can be classified into three configurations: Mirrored, distributed and sequential.

Mirrored configuration assumes that each display shows the same content [16]. The observations made by [17] in a lab study showed that a passerby first needs to notice the display and be motivated to interact. They recommend that a display should be placed so that, when people decide to interact, they are still in front of the display and do not have to walk back. Although, mirrored configuration increases the chance that a passerby sees 
the content, showing repetitive content can make the content obsolete for viewers, and consequently, decrease the display utility.

Distributed configuration occurs when separate or unrelated content is shown on the different screens.

Sequential configuration [18] corresponds to the assumption that a viewer will encounter a content sequence as they encounter a number of displays one after another, for example, while walking along a street. This allows additional [19], related or follow-on information to be viewed. For example, one display might show a news summary, while another one shows the corresponding images. Previous work has used such a configuration for dynamic wayfinding in crowded or complex spaces [20].

Although each configuration seems to have its own advantages, it is not clear what the effects of each configuration model are [16]. In addition, it is possible that there might be other more appealing configurations for particular scenarios and use cases. We therefore decided to identify and explore a wider range of configuration models.

\section{Configuration Models}

To come up with effective configuration models, we first needed to explore what possibilities exist for displaying content in a chained display configuration. We sought inspiration from currently deployed systems as well as possible future use cases of chained displays, which gave us a rough idea of what models might be appropriate. To get a broader view and validate our findings, we conducted a brainstorming session involving several staff and students of our research group. None of them had worked on public and semi-public displays. After introducing the chained displays and purpose of the session, we asked participants to think about apps for PDSs including the ones currently deployed and what might come in future (20min). We then asked how each of the apps can be configured for chained displays (40min). This session, together with some refinements, led to our final set of configuration models - Distributed, Mirrored, Parameter, Sequential, and Unity.

The models can be categorized into two subsets - unrelated and related. Unrelated configurations display content on each display which is not related to the content on other displays. The Distributed configuration is the only one belonging to this category. Related configurations are ones which display content on each display which is in some way related and coordinated with the content on the other displays. This includes Mirrored, Parameter, Sequential and Unity configuration models. A schematic representation of the configuration models is shown in Figure 1.

\subsection{Distributed}

The Distributed configuration displays unrelated content on each display and it is one of the most common setups today. This means that every display can work as a separate and independent unit and they require no adjustments for a chained display configuration. As shown in the schematic representation in Figure 1, the alphabet, the star symbol, the numbers and the car image represent different, unrelated applications. For example, one display could show weather information while another one displays news. 
Distributed

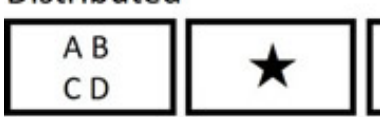

$1,2,3,4$

Mirrored

A

A

\begin{abstract}
A
\end{abstract}

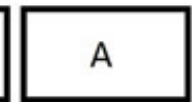

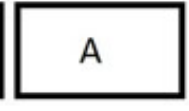

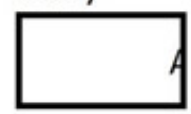

Sequential

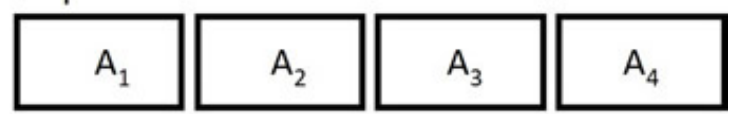

Unity

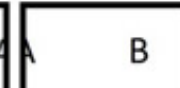

C

Parameter

C
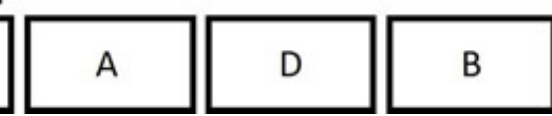

Figure 1: A schematic representation of the five configurations. A representation of the configurations using an article service is shown in Figure 7.

\subsection{Mirrored}

Another widely used configuration today, next to the distributed one, is the Mirrored configuration. It simply displays the same content on each display of the chained display configuration. Figure 1 highlights this by putting the same content (A) on each display.

One reason for the popularity of the mirrored configuration is probably its simplicity to set up. One just puts the same application on all the displays, resulting in presentation of the same content. Figure 2 shows an example of the mirrored configuration seen within a shopping center.

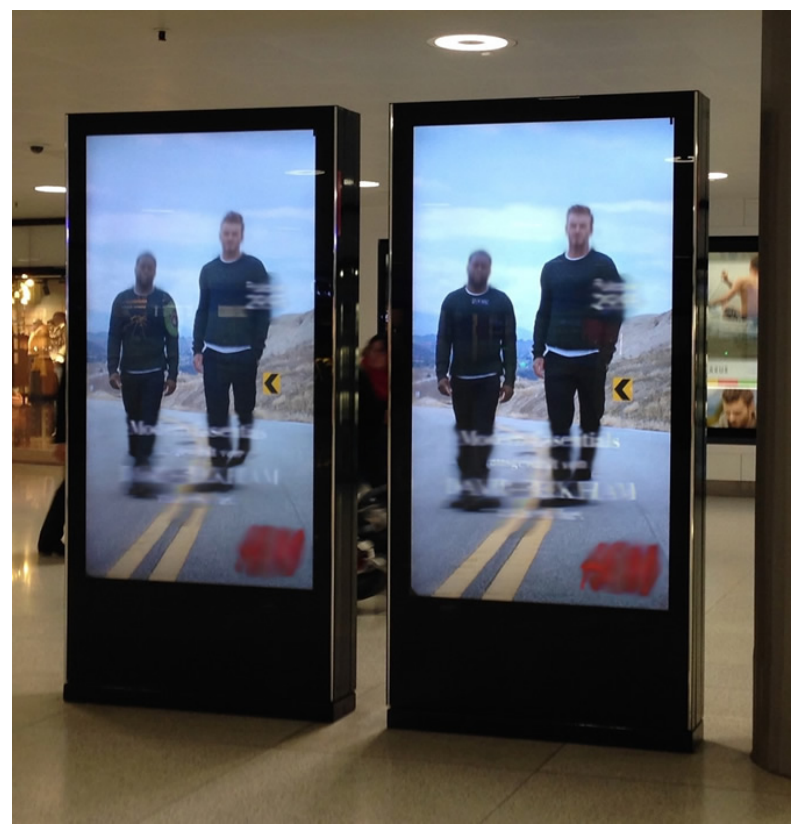

Figure 2: The Mirrored configuration used within a shopping center 


\subsection{Parameter}

The Parameter configuration publishes similar content on each display, but with a varying parameter. Importantly, there is no visible or particular order among the displays. The naming of this configuration stems from the observation that there is some parameter which changes from display to display. This could be, for example, weather information of different cities or news content separated by topic with each shown on a different display. In the schematic representation (see Figure 1), each letter stands for a different category of content. As the content is not associated with a particular order, people can approach the chained display configuration from any direction and still consume the content. Figure 3 shows a real-world example of such a configuration from our university. Two screens display the same menu of the cafeteria, but in different languages.

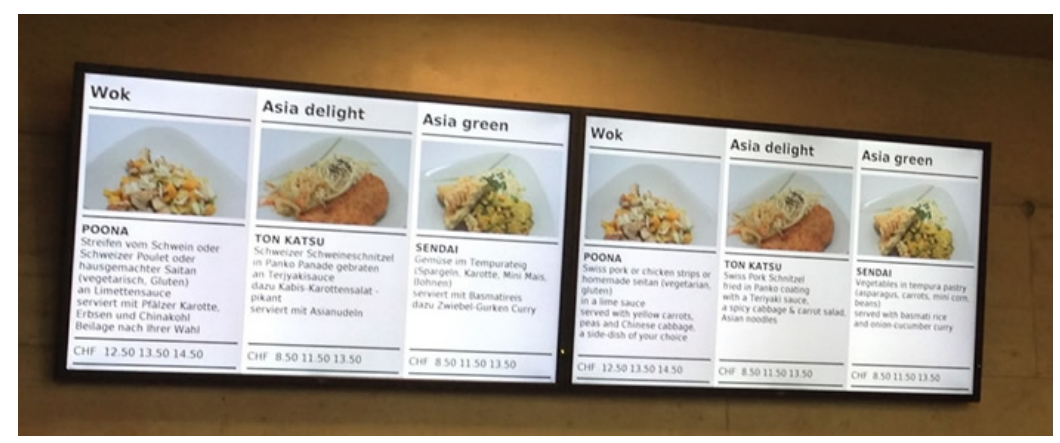

Figure 3: The Parameter configuration used to display the cafeteria menu in different languages

\subsection{Sequential}

As visualized in Figure 1, Sequential configuration spreads the content (A) in a sequence order over multiple displays. This creates a flow for the chained display configuration and guides the viewers through the content. This means that the displays should be visited by the viewer in a specific order for the best experience. Most often, this configuration is used to display lists of information which start on one display and continue on the next. It can also be used to provide follow up content to viewers. The first display might show the trailer of a movie and the others information about the actors. Sequential configuration can also be used to tell a part of an ongoing story on each display.

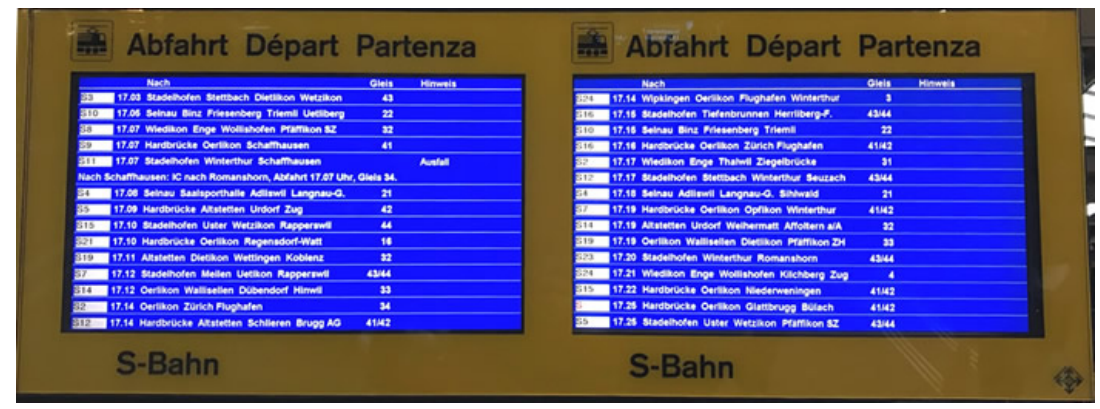

Figure 4: Two displays in a sequential configuration, displaying a list of departure times. 
One example of a continuous list over multiple displays can be seen in Figure 4 where the displays show a list of live departure times of trains.

\subsection{Unity}

Unity configuration treats the chained display configuration as if there were one wide display. Content can then be spread across this large virtual display without caring about the borders of the individual displays. In the schematic representation (Figure 1), the content of both A and D overlap the borders of two displays, while $\mathrm{B}$ and $\mathrm{C}$ are each on one display. As the displays are treated as a single large display, it may or may not happen that content overlaps the border. Figure 5 shows an example of the Unity configuration with an advertisement displayed over five screens that are encountered in sequence while walking in an airport.

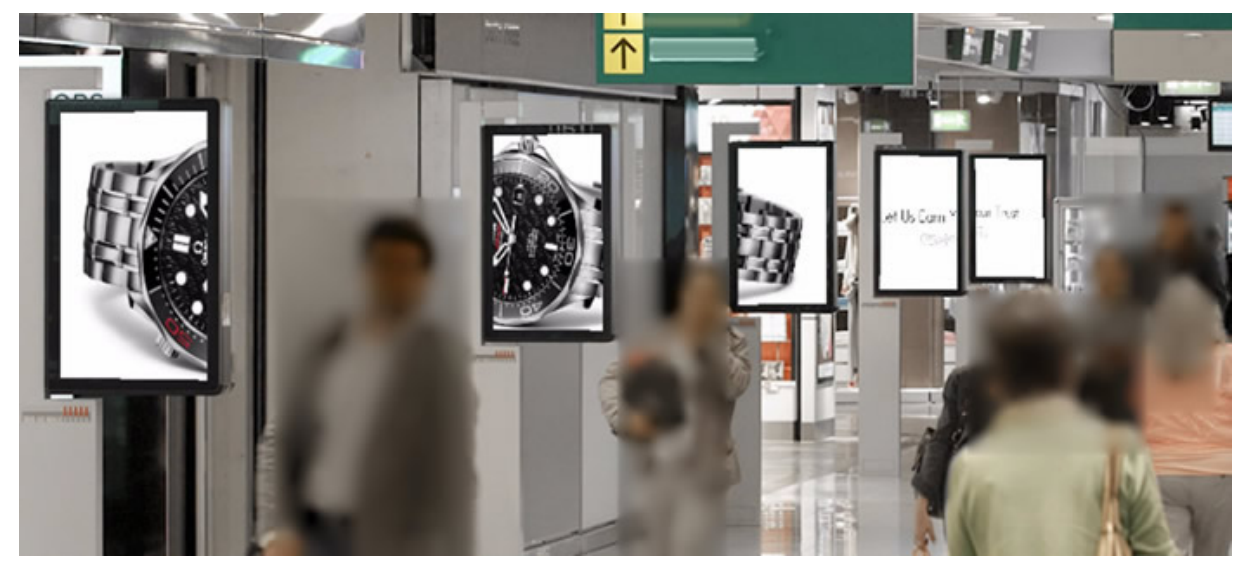

Figure 5: Five displays in a Unity configuration at Airport

\section{Implementation}

To implement and test the configurations, we extended ScreenPress which is a platform developed for the rapid prototyping of PDS [2]. ScreenPress is based on a client-server architecture and allows the system to be extended using a plugin mechanism. Further, it separates the concepts of content and visualization which enables the content of services to be visualized using the configuration models independent of the content or the service. We extended the platform by writing a plugin to add support for managing chained display configurations.

Figure 6 shows part of the ScreenPress metamodel and its relation to our extension configuration class. Configuration model refers to the configuration models mentioned earlier. Dashed lines represent the connection between the extended subpart and the rest of the ScreenPress metamodel. A visualization refers to how a content item should be displayed on the connected displays. Each visualization can provide one or more configurations. Each service in a given context can then be connected to one of those configurations, defining how this service will be shown on the displays. 


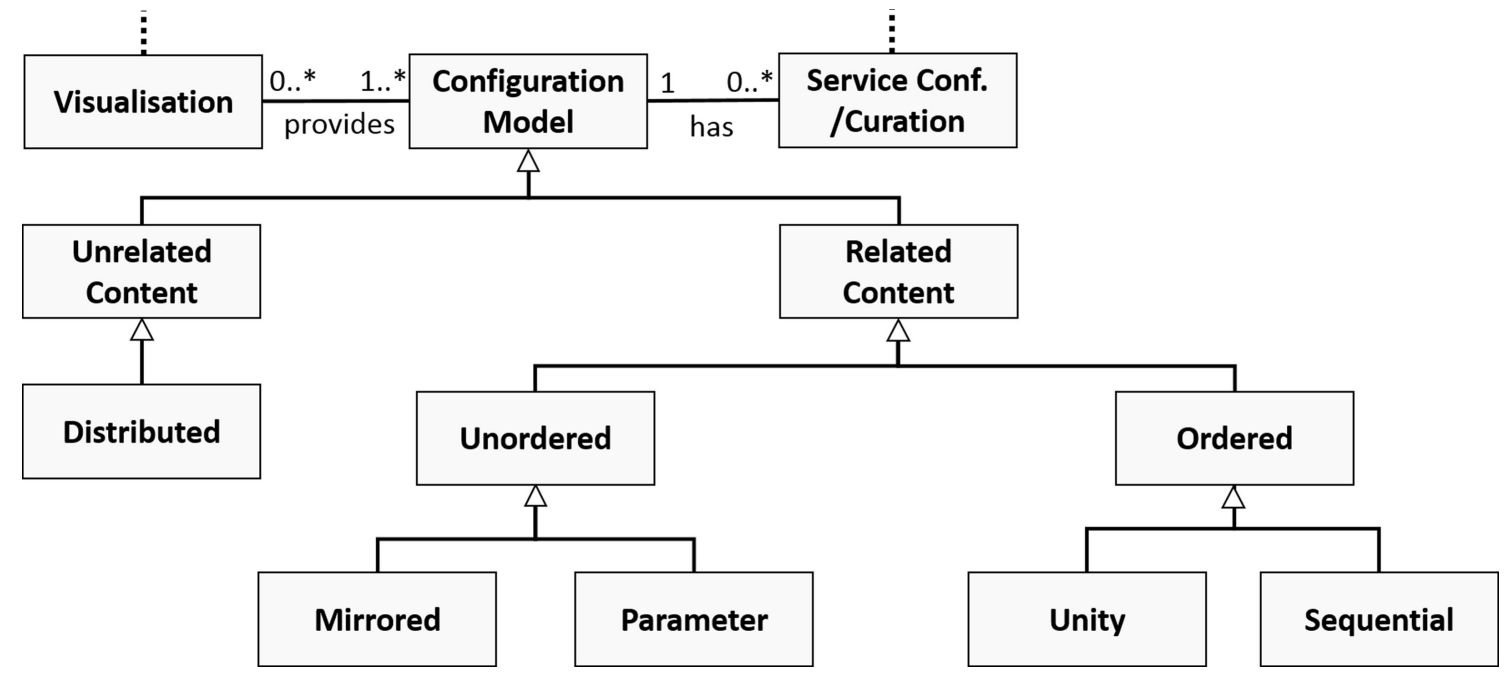

Figure 6: The metamodel of the configuration models

A service provides content from any kind of data source, but does not influence the visualization of the content. The service provides the content in the JSON format shown in Code Snippet 1. The service data is then available to be used for visualization.

The outermost structure in the code snippet 1 is an array of categorized elements (e.g. weather cities). A category element may have multiple content elements in its data array, typically the filter objects (e.g. different weather forecasts). Each such content element has a sequenceNumber which defines a sequence over all the content elements. This enables the template to correctly display the content in a sequential configuration. The innermost nesting represents the individual data elements (e.g. title, weather image) of a content element. One example of such a data element array would be a title, a feature image and a description text.

A template is responsible for the visual style of the displays. It is an HTML document which uses HTML, CSS and JavaScript to parse and render the data it gets from the services.

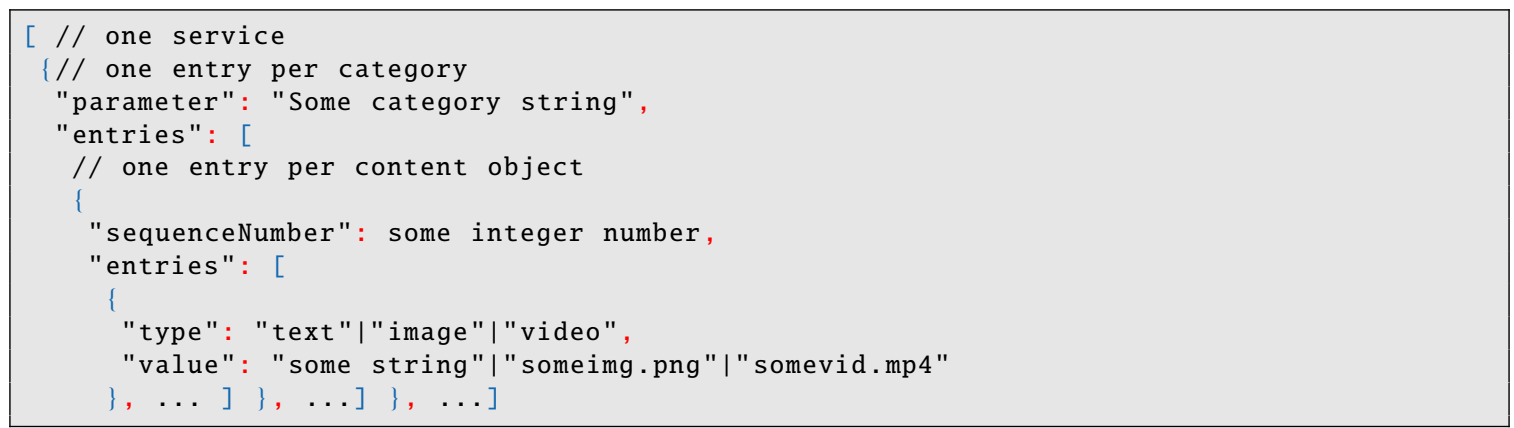

Code Snippet 1: Structure of the data for each service

Each template also defines which configurations it provides. The platform dashboard will then allow the user to choose one of the configurations provided by the template for each activated service.

Each public display loads a URL which serves the activated template to the display. 
Through an active connection to the server, the template receives all data updates for the services as well as other updates such as template changes or changes to the chained display configuration.

\subsection{Prototypes}

We developed two sample prototypes to validate the extension of our system and its possible use in an actual public display deployment - a weather service and an article service.

The weather service shows the weather forecast for the cities specified in the ScreenPress configuration. The service data provides a title with the name of the city and the service uses the weather data to get the forecast for the next three days including today.

\section{Distributed}
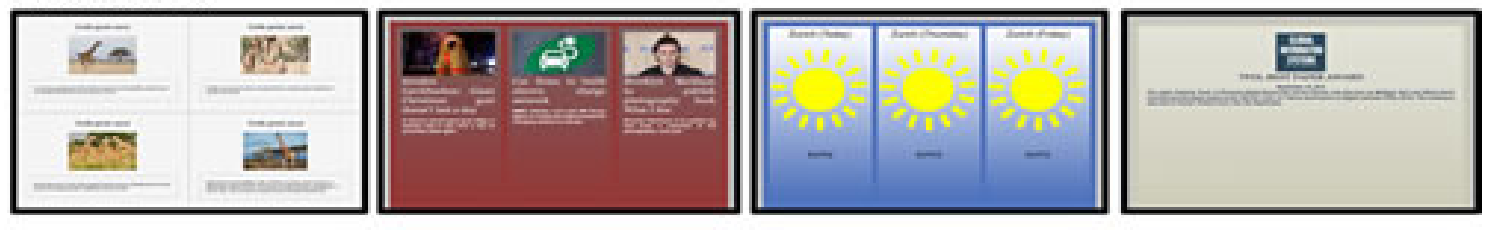

\section{Mirrored}
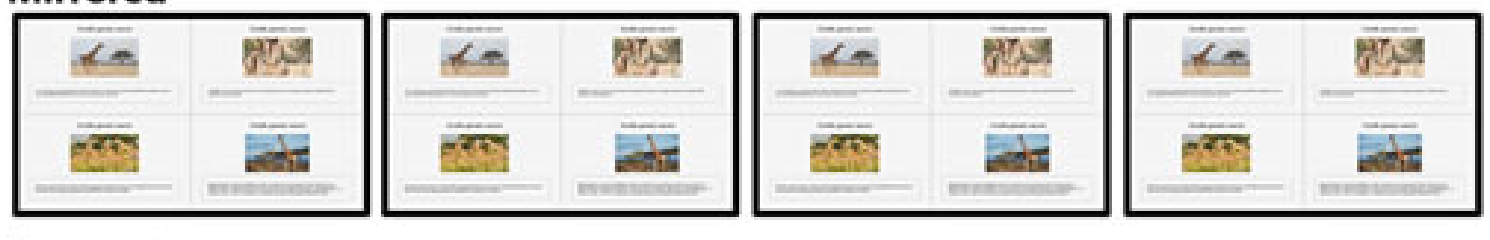

\section{Parameter}
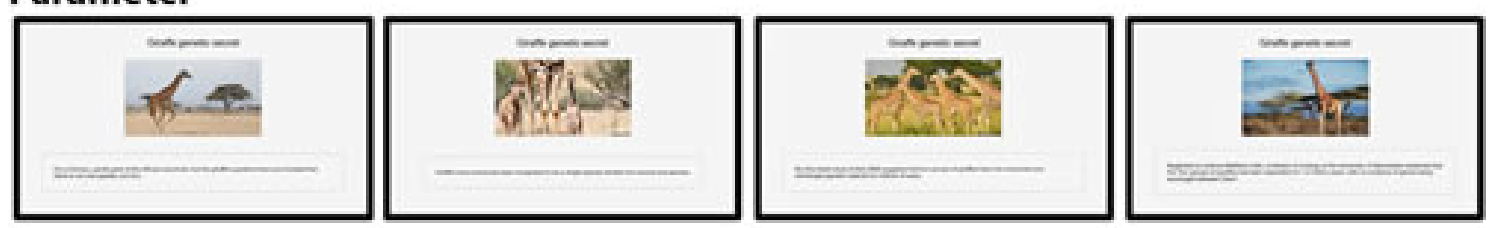

\section{Sequential}
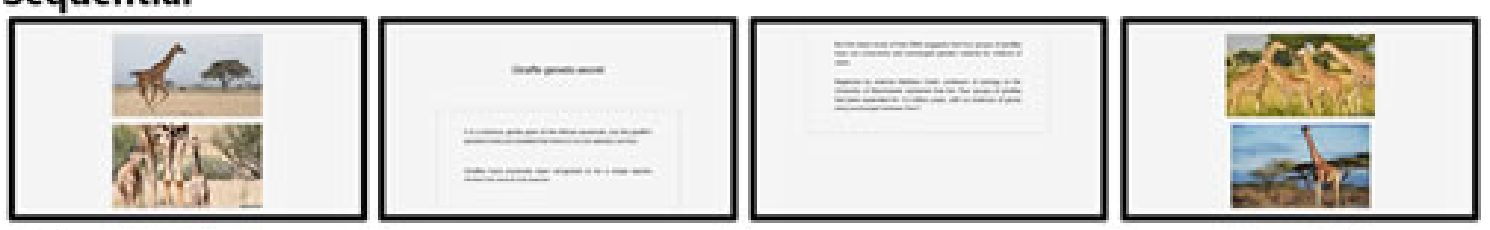

\section{Unity}
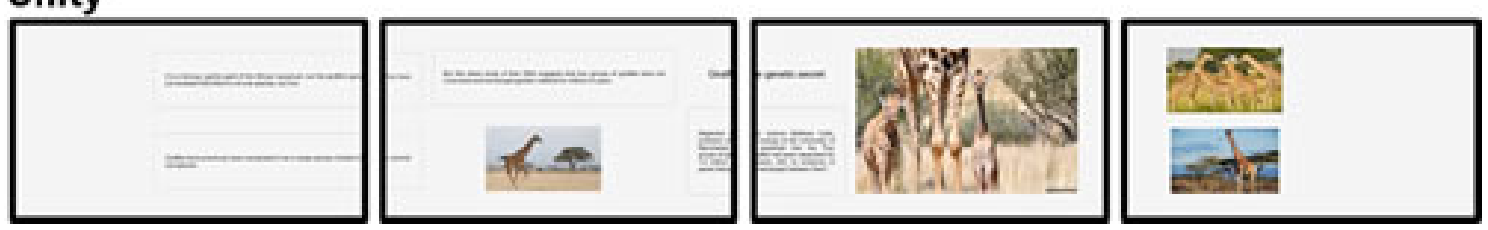

Figure 7: An overview of the article service in all the configurations.

The article service enables ScreenPress users to display articles on the PDS. Figure 7 provides an overview of all the configurations with the article service and a template. The Distributed and Mirrored configurations show all four parts of the article on a single screen. The Parameter configuration puts each independent part of the article on a separate 
display, which results in one image and one paragraph on each display. The Sequential configuration aligns the four parts of the article next to each other while also separating images and text. This gives the content a flow from left to right. The Unity configuration puts the title of the article in the middle and aligns the text pieces and images around it, resulting in multiple text fragments ranging over multiple displays. For this figure we used the giraffe article which was one of five articles used in the user study.

\section{User Study}

The goal of the user study was to explore the benefits and deficits of different configurations with respect to usability and viewer engagement.

We opted for a controlled lab study, as for the anticipated measurement of user experience on configuration models, a highly controllable environment was required to minimize the external influences (e.g., other passersby, environmental conditions) as it is discussed for public displays in $[21,22]$.

\subsection{Participants}

We recruited 21 participants (13 males; age: 23-55 (median=26)) with different levels of education (4 High school, 5 Bachelors, 9 Masters, and 3 PhDs). Participants were recruited at our university and also from our social circle using the snowball sampling method. All the participants had normal or corrected-to-normal eyesight.

\subsection{Methodology and Procedure}

The study was done individually in our lab's meeting room (size $8 x 8 \mathrm{~m}^{2}$ ). We had an identical setup for all the configurations and used the flat-display setup which was found to have the strongest effect on viewers [12]. To present our configurations, we used four 32" displays placed in landscape mode (see Figure 8). Displays were aligned next to each other at eye-level. For the study, we used the developed article service. We had five articles; each consisted of 6 to 9 facts in approximately 100 words and four images. All of the articles were shortened versions of articles from bbc.com, a British news website. The types of contents used include science ${ }^{1}$, society ${ }^{2}$, nature ${ }^{3}$, sport $^{4}$, and culture ${ }^{5}$. For each participant, the order of the configurations was randomized. Moreover, the pairing of configurations and articles was randomized (without replacement) to ensure that the result for a configuration was not influenced by the content of a particular article. The Distributed configuration required displaying different unrelated contents on each display. To present this configuration in a clear distinguishable manner, in addition to the article service, we used the weather service as well as two other services called lucky number and group information services. Each service was individually shown on one of the displays.

\footnotetext{
${ }^{1}$ http://www.bbc.com/news/science-environment-37311716

${ }^{2} \mathrm{http}: / / \mathrm{www} . \mathrm{bbc} . \mathrm{com} /$ capital/story/20160325-the-surprising-benefits-of-a-mid-career-break

${ }^{3}$ http://www.bbc.com/earth/story/20160823-the-real-origin-of-north-americas-wolves

${ }^{4} \mathrm{http}: / / \mathrm{www} . \mathrm{bbc} . \mathrm{com} / \mathrm{sport} / \mathrm{cricket} / 37365637$

${ }^{5}$ http://www.bbc.com/travel/story/20160912-the-treacherous-road-with-318-turns
} 


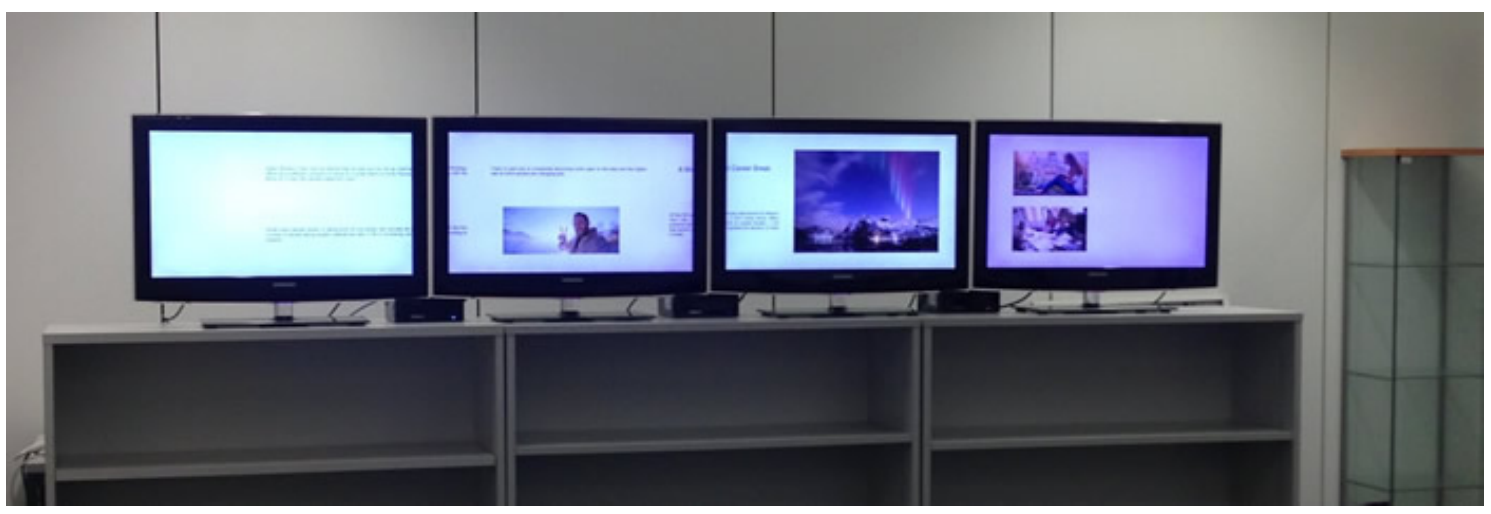

Figure 8: The study setup.

Before gathering demographic information of the participants, we gave each participant a short introduction about public and semi-public displays, chained displays, and what a configuration is. We also told the participants the purpose and process of the study. We did not introduce the participants to the individual configurations, as we did not want to influence them on their attitude towards each of the configurations. For each of the configurations, the task was to read the displayed article. Participants were allowed to freely walk around and complete the task without any time limit. The reading task ensured that the participants spend enough time with the displays to get to know the individual configurations. The entire user study took about 45 minutes for each participant.

The participants used the configurations one after the other. Following each reading task, participants were asked to fill out (1) System Usability Scale (SUS) questionnaire [23]; and (2) our questions designed based on the User Engagement Scale (UES) guideline [24, 25] shown in Table 1. At the end of the study, we also asked participants several semistructured and open-ended questions about their previous experience with the configuration models and public displays. These questions also included what factors participants think can influence the performance of the configurations and how the configurations differ from each other with respect to those factors.

The SUS consisted of 10 questions resulting in a single measure of usability that is between 0 and 100. We used the SUS score to compare the usability of configuration models. Above $68 \%, 74 \%$, and $80.3 \%$ usability scores are considered as average (grade C), good (grade B), and excellent (grade A) usability performance, respectively [23,26].

The UES provided six specific dimensions of viewer engagement as shown in Table 1. The questions corresponding to each dimension were on a 5 point Likert scale, mapped into a 0 to 4 score range. Focused Attention describes up to which degree the system attains the user's full attention. Felt Involvement describes how involved the user felt with the system. Novelty measures the viewer's curiosity and degree of interest. Endurability evaluates the system's overall success, willingness of recommendation, and whether the viewer would use the system again. Aesthetic Appeal measures the visual quality of the system. Perceived Usability reflects the user's satisfaction with the system.

One-way repeated measures ANOVA and post-hoc analysis were performed to determine statistical differences between the SUS score of different configurations.

To compare the configurations with respect to the UES dimensions, we combined the results of the questions related to each dimension by adding up the received scores. We 
performed a Friedman test to check whether there is a significant difference between the configurations in terms of the scores in each UES dimension. If the outcome of this test was significant, we performed post hoc analysis by performing pairwise comparisons using Wilcoxon Signed Rank Tests to check where the differences between the configurations are, and adjusted the calculated p-values based on Bonferroni multiplicity correction as $p$-value ad justed $=p$-value original $\times 10$ (total number of pairwise comparisons). Accordingly, the reported $\mathrm{p}$-values for this comparisons are adjusted p-values. We set the minimum significance level at 0.05 .

\subsection{Results}

The results of pairwise comparisons of the SUS scores per configuration are represented in Figure 9. There was a significant effect of configurations on the SUS scores $(F(4,80)=$ 21.6, $\left.p<0, \eta_{p}^{2}=.52\right)$. Both Parameter and Sequential scored excellent and are not significantly different from each other. The SUS of Distributed and Mirrored both score above average, while both statistically score worse than Parameter. The Mirrored SUS score is also significantly worse than Sequential. The Unity SUS score is below the average and it is significantly worse than all the other configurations.

Significant results of post hoc analysis for comparison of configurations in all six UES dimensions are given in Table. 2.

For the focused attention dimension, Parameter configuration scores best and is significantly better than the Distributed configuration $(p=0.03)$. However, there is no statistically

Table 1: UES Dimensions and questions; *reverse coded items.

\begin{tabular}{ll}
\hline UES Dimension & Question \\
\hline \multirow{2}{*}{ Focused Attention } & $\begin{array}{l}\text { I lost myself in this viewing experience. } \\
\text { I was so involved in my viewing task that I lost track of time. } \\
\text { I blocked out things around me when I was looking at the displays. } \\
\text { I was absorbed in my viewing task. }\end{array}$ \\
\hline Felt Involvement & $\begin{array}{l}\text { I felt involved in this viewing task. } \\
\text { This viewing experience was fun. }\end{array}$ \\
\hline \multirow{2}{*}{ Novelty } & $\begin{array}{l}\text { I continued to look at the displays out of curiosity. } \\
\text { The content of the displays incited my curiosity. }\end{array}$ \\
\hline \multirow{2}{*}{ Endurability } & $\begin{array}{l}\text { I felt interested in my viewing task. } \\
\text { I would recommend looking at those displays to my friends and family. }\end{array}$ \\
\hline \multirow{2}{*}{ Aesthetic Appeal } & $\begin{array}{l}\text { This viewing configuration is attractive. } \\
\text { I liked the graphics and images used on this customisation. } \\
\text { The screen layout of this customisation was visually pleasing. }\end{array}$ \\
\hline \multirow{2}{*}{ Perceived Usability } & $\begin{array}{l}\text { I felt frustrated while looking at the displays.* } \\
\text { Looking at the displays was mentally taxing.* } \\
\text { This viewing experience was demanding.* }\end{array}$
\end{tabular}




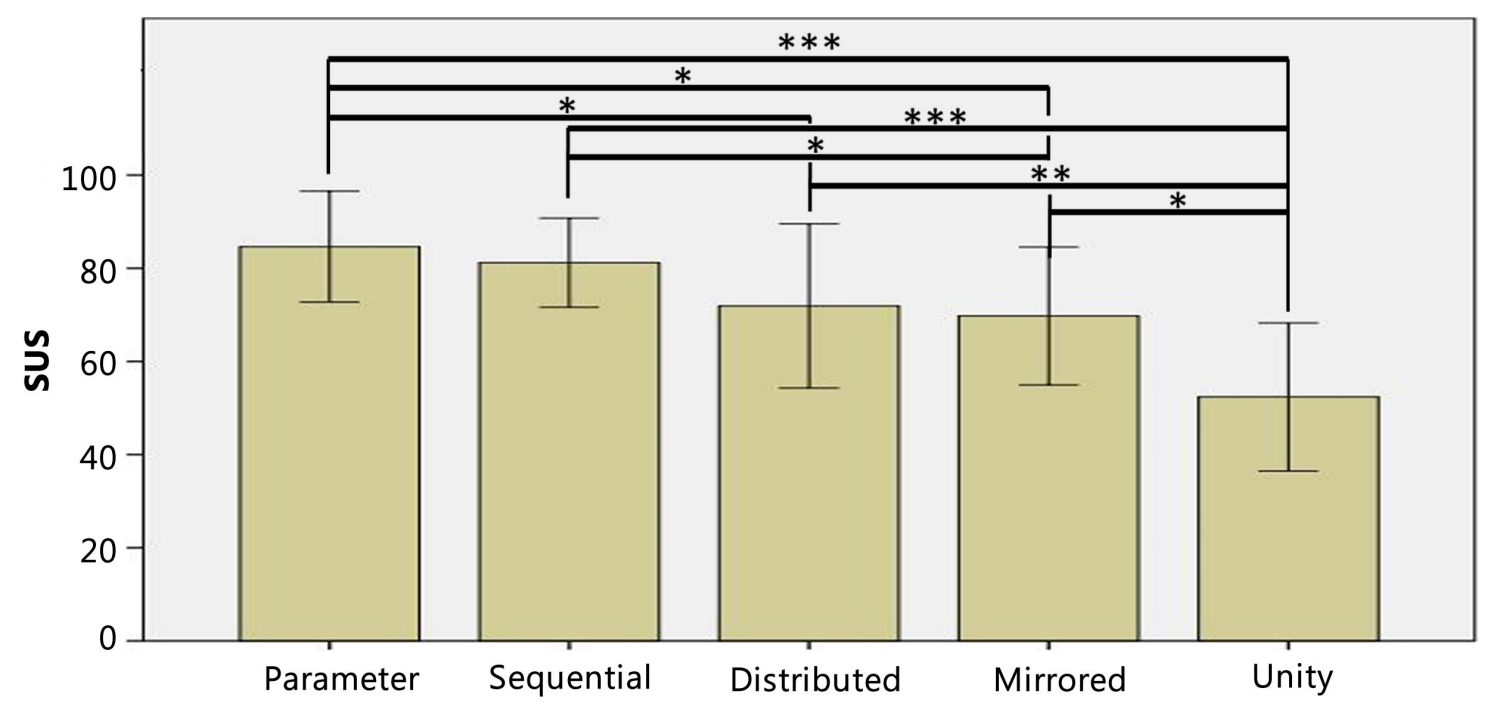

Figure 9: Mean and standard deviation of SUS scores for each configuration. The *,**, $* * *$ mark significant differences between configurations and correspond to $\mathrm{p}<0.05, \mathrm{p}<$ 0.005 , and $\mathrm{p}<0.001$, respectively.

significant difference between other pairwise comparisons. In the felt involvement dimension, both Parameter $(p=0.01)$ and Sequential $(p=0.01)$ score significantly better than Unity. There is a statistically significant difference in the novelty dimension between Sequential and Mirrored in favour of Sequential $(p=0.03)$. In the same dimension, Parameter is significantly better than Unity $(p=0.01)$. In the endurability dimension, both Parameter $(p<0.01)$ and Sequential $(p<0.01)$ are significantly better than Unity. There is a statistically significant difference effect in the aesthetic appeal dimension between Parameter and Unity $(p<0.01)$ as well as Sequential and Unity $(p<0.01)$ in favour of Parameter and Sequential. For the perceived usability dimension, Parameter $(p<0.01)$, Sequential $(p<0.01)$, Distributed $(p<0.01)$, and Mirrored $(p<0.01)$ are all statistically better than the Unity configuration.

We asked participants how often they had encountered the different configurations before the study, and the most prominent were Mirrored and Distributed.

\subsubsection{Qualitative Feedback}

The feedback provided as comments gave us a better insight into the configuration models.

One participant preferred Distributed as people do not need to switch between screens.". However, some participants mentioned that a visual commonality between the displays is important. This allows viewers to faster find their content of interest. Further, the possibility of consuming the content on the move was also appreciated.

The Mirrored configuration was considered as "..super boring" and "..waste of space", since the same content is displayed on all screens. For the Parameter configuration, some suggested having a "visual hint, so it's clear that the screens show different content from afar". For Parameter, it was suggested that there should be "No broad margin between the displays". Separation of text and images on different displays was appreciated in Sequential. However, some participants were concerned that "reading text without pictures on large displays is demanding". An indication of the direction of the text on displays was 
Table 2: Statistical comparison of configuration models on each UES dimension; *adjusted p-value. According to [27] criteria the effect size above: $0.1=$ small effect, $0.3=$ medium effect, $0.5=$ large effect.

\begin{tabular}{|c|c|c|c|c|c|c|c|c|c|}
\hline \multirow{2}{*}{$\begin{array}{l}\text { UES Dimensions } \\
\text { Dimension [max score] }\end{array}$} & \multicolumn{5}{|c|}{ Conf. [median] } & \multicolumn{4}{|c|}{ Results } \\
\hline & Par & Seq & Uni & Dis & Mir & Outcome & Z-Value & p-Value* & Effect Size \\
\hline Focused Attention [16] & 9 & 10 & 8 & 7 & 8 & Par $>$ Dis & -2.854 & $=0.03$ & 0.44 \\
\hline \multirow{2}{*}{ Felt Involvement [8] } & \multirow{2}{*}{6} & \multirow{2}{*}{6} & \multirow{2}{*}{3} & \multirow{2}{*}{4} & \multirow{2}{*}{4} & Par $>$ Uni & 3.045 & $=0.01$ & 0.47 \\
\hline & & & & & & Seq $>$ Uni & 3.146 & $=0.01$ & 0.485 \\
\hline \multirow{2}{*}{ Novelty [12] } & \multirow{2}{*}{9} & \multirow{2}{*}{9} & \multirow[b]{2}{*}{7} & \multirow[b]{2}{*}{9} & \multirow{2}{*}{6} & Seq $>$ Mir & -2.836 & $=0.03$ & 0.438 \\
\hline & & & & & & Par $>$ Uni & 2.973 & $=0.01$ & 0.459 \\
\hline \multirow{2}{*}{ Endurability [8] } & \multirow{2}{*}{6} & \multirow{2}{*}{6} & \multirow{2}{*}{3} & \multirow{2}{*}{4} & \multirow{2}{*}{4} & Par $>$ Uni & 3.393 & $<0.01$ & 0.524 \\
\hline & & & & & & Seq $>$ Uni & 3.44 & $<0.01$ & 0.531 \\
\hline \multirow{2}{*}{ Aesthetic Appeal [12] } & \multirow{2}{*}{9} & \multirow{2}{*}{9} & \multirow[b]{2}{*}{4} & \multirow{2}{*}{7} & \multirow[b]{2}{*}{6} & Par $>$ Uni & 3.782 & $<0.01$ & 0.584 \\
\hline & & & & & & Seq $>$ Uni & 3.659 & $<0.01$ & 0.565 \\
\hline \multirow{4}{*}{ Perceived Usability [16] } & \multirow{4}{*}{13} & \multirow{4}{*}{13} & \multirow{4}{*}{5} & \multirow{4}{*}{12} & \multirow{4}{*}{11} & Par $>$ Uni & 3.884 & $<0.01$ & 0.599 \\
\hline & & & & & & Seq $>$ Uni & 4.021 & $<0.01$ & 0.62 \\
\hline & & & & & & Dis $>$ Uni & 3.685 & $<0.01$ & 0.569 \\
\hline & & & & & & Mir $>$ Uni & 3.552 & $<0.01$ & 0.548 \\
\hline
\end{tabular}

suggested as a possible improvement. One positive feedback for Unity was the "motivation to follow the information and move to different displays to find the rest of the information."

\section{Discussion}

The results of the user study showed statistical differences between the configuration models. The results revealed that the performance of configurations differs with respect to different factors that should be taken into account when deploying a chained display configuration. A decision on configuration can be based on the dimensions that are most important for the stakeholders of a PDS.

The statistical analysis of SUS, UES and a review of qualitative feedback showed that Parameter and Sequential were highly liked since they make good use of the available display space and provide a clear separation of the content for each display. In today's chained display configurations, Distributed and Mirrored are still generally good choices, but, in most situations, Parameter and Sequential would be a better fit. Unity is in general disliked by the viewers, but has some valid use cases. The result of Unity is in line with the observation in a previous study [28] that revealed the users were discouraged to make the content span multiple displays because of the visible gap between individual monitors. Alternatively, users used additional monitors to separate content belonging to different tasks.

In the dimension Focused Attention, the configuration Parameter focuses viewer attention more than Distributed. Using Distributed, retaining the attention is unlikely as the viewer needs to be interested in all of the content. With Mirrored, the viewers focus their attention on only one of the displays and even can get demotivated to focus on other displays when they see that they just show the same content. 
In Felt Involvement dimension, Parameter and Sequential score better than Unity. With the Parameter and the Sequential configurations, people get drawn into the viewing experience. Users agreed that the viewing experience was especially fun when exposed to those two configurations. A potential reason for the shortcoming of Unity is the fact that it is maybe mentally taxing to read over the display borders which opens up viewers' mind to distractions. The Distributed and Mirrored configurations were scored average for the Felt Involvement. This might be because people have problems being driven into the viewing experience when each display shows completely different or exactly the same content.

With respect to Novelty dimension, Parameter and Sequential feel most novel. The fact that with these configurations the displays share a common topic while presenting new or follow up content supports a viewer's curiosity. Although, many people agreed on the Novelty of Unity, it is still significantly worse than Parameter and does not have a significant difference from any other configuration. The low Novelty score for the Mirrored configuration can be because the viewers lose interest when they realize that other displays show the same content.

Concerning the Endurability dimension of UES, with Parameter and Sequential, viewers are more likely to return to the displays. These configurations are also more likely to be recommended to others. With Unity, only a few saw their viewing experience as a success. While reading over the seams is to blame, it may also be difficult for people to reconstruct all the displays in their mind, especially for Unity. Reading is much easier for the other configurations than Unity as they have a clear separation of the content per display.

In terms of Aesthetic Appeal, the screen layout was most pleasing to viewers in the Sequential and Parameter configurations, whereas Mirrored and Distributed scored neutral. This can be because Distributed configuration missed a visual common ground between the displays and the mirrored configuration had too much content packed on each display. Good graphical design can improve Unity's Aesthetic Appeal, but it was generally not considered attractive, especially if there are large borders or spaces between the displays.

For Perceived Usability dimension, all configurations except Unity were perceived as highly usable. This finding is in line with our results of SUS. For this dimension, the results are clearly distinct between the unity configuration and the other configurations. The Unity configuration was the only one which was reported to be frustrating to look at and to be mentally taxing. Participants said that this was because of the borders between the displays which made it challenging to read text which crossed two displays.

\section{$7 \quad$ Limitations and Future Work}

While the lab study provided a high degree of control and precise results, at the same time provided a lower degree of ecological validity. In order to ecologically confirm our lab finding, future research in a public setting would be necessary.

In our study, we used a particular set of content types. Different content types may be more suitable for a certain type of configuration. Therefore, a more in-depth comparative study of the combinations of different content and configuration types is needed.

From the analysis of participants' responses to the semi-structured and open-ended questions, we identified other factors that may influence the performance of the configurations which should be considered. From the participants responses, we generated the list of factors, grouped them together according to their conceptual similarity, and integrated 
the hypothetical answers for each of the identified factors. We believe these factors and the associated hypotheses are interesting aspects that need to be evaluated in the future.

The Distance Between the Displays: Which configuration is preferable depends on the distance between the displays relative to the viewer's position. If the viewer can easily see and read the content of two displays at the same time from their position, the mirrored configuration does not make sense. It does, however, make sense to use the mirrored configuration if the displays are far away from each other and the viewer should be reminded of the content. For the Distributed and Parameter configurations, the distance between the displays is not relevant. The appropriate distance between the displays plays a major role, especially when using Sequential or Unity configurations where they might have to remember the content of the previously viewed display.

Order in Which Viewers Encounter the Displays: If the sequence in which the viewers will encounter the displays is unknown, Parameter, Mirrored or Distributed are better than Sequential and Unity, as they do not impose an order on content. If the order is not random, but either left to right or right to left, Unity might be a good choice. For Sequential, the order in which the displays will be encountered must be known beforehand to properly display content.

The Speed of Viewer Movement: If viewers pass the displays very quickly, Mirrored is suitable as the chance to grab the content is higher. If the speed is normal or slow, other configurations might be more suitable as viewers get new content on each display.

The Content on the Displays The Mirrored configuration is not suitable for a large amount of content, as it leads to small font and image sizes. Distributed configuration needs the content to be unrelated and each piece of content should not be large either as it has to fit on one display. With the Sequential and Parameter configurations, the content should be separable. If this is not the case, Unity should be used.

Viewer Expectations: Knowing a viewer's expectations and reacting to them can influence viewer engagement. Fulfilling negative expectations, or not living up to positive ones, leads to boredom on the part of viewers and will prevent them from returning to the displays at another time. The riskiest configuration then is Mirrored. If people expect fresh content and become irritated by repetitive content, the screens may be ignored in the future.

For Sequential and Parameter configurations, it is important to provide hints that there are other displays showing related content. Otherwise, viewers might miss the content they are looking for. The Unity configuration provides such a hint by design.

The Number of Viewers A large number of viewers can occlude each other's view of the displays. If it is important to provide the content to everyone, the Mirrored configuration is most suited. Otherwise, Distributed and Parameter are a good choice as viewers can spread themselves among the displays. In the case of an individual viewer in front of the displays, all configurations except the Mirrored one can be suitable.

\section{Conclusion}

We have presented possible configuration models for chained displays and reported on a user study. We compared the configurations effectiveness and evaluated whether there are differences between them. The study provided better insights into the effect of the different configurations in terms of usability and user engagement. We found that the configurations differ in terms of usability and also with respect to different dimensions 
of user engagement. The results revealed that the most commonly used configurations, Distributed and Mirrored, are suboptimal and improvements can be made by using other configurations such as Parameter and Sequential. In the future, we want to extend our investigations to explore the configuration effects in the wild as well as taking into account interaction with chained displays.

\section{References}

[1] Nigel Davies, Marc Langheinrich, Rui Jose, and Albrecht Schmidt. Open Display Networks: A Communications Medium for The $21^{\text {st }}$ Century. Computer, (5):58-64, 2012 .

[2] Amir E. Sarabadani Tafreshi and Moira C. Norrie. ScreenPress: A Powerful and Flexible Platform for Networked Pervasive Display Systems. In Proc. 6th ACM Intl. Symposium on Pervasive Displays (PerDis), pages 13:1-13:8. ACM, 2017.

[3] Amir E. Sarabadani Tafreshi, Sara C. Sarabadani Tafreshi, and Amirehsan Sarabadani Tafreshi. TiltPass: Using Device Tilts As an Authentication Method. In Proceedings of the 2017 ACM International Conference on Interactive Surfaces and Spaces (ISS), pages 378-383. ACM, 2017.

[4] Jörg Müller, Dennis Wilmsmann, Juliane Exeler, Markus Buzeck, Albrecht Schmidt, Tim Jay, and Antonio Krüger. Display blindness: The Effect of Expectations on Attention Towards Digital Signage. In Pervasive Computing, pages 1-8. Springer, 2009.

[5] Jörg Müller, Robert Walter, Gilles Bailly, Michael Nischt, and Florian Alt. Looking Glass: A Field Study on Noticing Interactivity of a Shop Window. In Proceedings of the SIGCHI Conference on Human Factors in Computing Systems, pages 297-306. ACM, 2012.

[6] Amir E. Sarabadani Tafreshi, Kim Marbach, and Moira C. Norrie. Proximity-Based Adaptation of Web Content on Public Displays. In Web Engineering, pages 282-301. Springer, 2017.

[7] Amir E. Sarabadani Tafreshi, Kim Marbach, and Gerhard Tröster. Proximity-Based Adaptation of Content to Groups of Viewers of Public Displays. In International Journal of Ubiquitous Computing (IJU), pages 1-9, 2018.

[8] Amir E. Sarabadani Tafreshi, Adrian Wicki, and Gerhard Tröster. RDSpeed: Development Framework for Speed-Based Adaptation of Web Content on Public Displays. In 26th International Conference in Central Europe on Computer Graphics, Visualization and Computer Vision (WSCG). WSCG, 2018.

[9] Florian Ott, Andrea Nutsi, and Peter Lachenmaier. Information Ergonomics Guidelines for Multi-User Readability on Semi-Public Large Interactive Screens. In Proc. of the Workshop Information Ergonomics at iKnow Conference, 2014. 
[10] Peter Peltonen, Esko Kurvinen, Antti Salovaara, Giulio Jacucci, Tommi Ilmonen, John Evans, Antti Oulasvirta, and Petri Saarikko. It's Mine, Don't Touch!: Interactions at a Large Multi-Touch Display in a City Centre. In Proceedings of the SIGCHI Conference on Human Factors in Computing Systems (CHI), pages 1285-1294. ACM, 2008.

[11] Amir E. Sarabadani Tafreshi, Andrea Soro, and Gerhard Tröster. Automatic, Gestural, Voice, Positional, or Cross-Device Interaction? Comparing Interaction Methods to Indicate Topics of Interest to Public Displays. In Frontiers in ICT. Frontiers, 2018.

[12] Maurice Ten Koppel, Gilles Bailly, Jörg Müller, and Robert Walter. Chained Displays: Configurations of Public Displays Can Be Used to Influence Actor-, Audience-, and Passer-By Behavior. In Proc. SIGCHI Conference on Human Factors in Computing Systems (CHI), pages 317-326. ACM, 2012.

[13] Antonio Gomes and Roel Vertegaal. PaperFold: Evaluating Shape Changes for Viewport Transformations in Foldable Thin-Film Display Devices. In Proceedings of the Ninth International Conference on Tangible, Embedded, and Embodied Interaction (TEI), pages 153-160. ACM, 2015.

[14] Jason Alexander, Andrés Lucero, and Sriram Subramanian. Tilt Displays: Designing Display Surfaces With Multi-Axis Tilting and Actuation. In Proceedings of the 14th International Conference on Human-computer Interaction with Mobile Devices and Services (MobileHCI), pages 161-170. ACM, 2012.

[15] Ken Hinckley, Morgan Dixon, Raman Sarin, Francois Guimbretiere, and Ravin Balakrishnan. Codex: A Dual Screen Tablet Computer. In Proceedings of the SIGCHI Conference on Human Factors in Computing Systems (CHI), pages 19331942. ACM, 2009.

[16] Umar Rashid, Miguel A. Nacenta, and Aaron Quigley. Factors Influencing Visual Attention Switch in Multi-display User Interfaces: A Survey. In Proceedings of the 2012 International Symposium on Pervasive Displays (PerDis), pages 1:1-1:6. ACM, 2012.

[17] Florian Alt. A Design Space for Pervasive Advertising on Public Displays. PhD thesis, 2012.

[18] Constantin Taivan. Web-based Applications for Open Display Networks. PhD thesis, 2014.

[19] Tero Jokela, Jarno Ojala, and Thomas Olsson. A Diary Study on Combining Multiple Information Devices in Everyday Activities and Tasks. In Proc. 33rd ACM Conf. on Human Factors in Computing Systems (CHI), pages 3903-3912. ACM, 2015.

[20] Jorgos Coenen, Niels Wouters, and Andrew Vande Moere. Synchronized Wayfinding on Multiple Consecutively Situated Public Displays. In Proceedings of the 5th ACM International Symposium on Pervasive Displays (PerDis), pages 182-196. ACM, 2016. 
[21] Florian Alt, Stefan Schneegaß, Albrecht Schmidt, Jörg Müller, and Nemanja Memarovic. How to evaluate public displays. In Proceedings of the 2012 International Symposium on Pervasive Displays, pages 17:1-17:6. ACM, 2012.

[22] Gilbert Beyer, Florian Alt, Jörg Müller, Albrecht Schmidt, Karsten Isakovic, Stefan Klose, Manuel Schiewe, and Ivo Haulsen. Audience Behavior Around Large Interactive Cylindrical Screens. In Proc. SIGCHI Conference on Human Factors in Computing Systems (CHI), pages 1021-1030. ACM, 2011.

[23] Jeff Sauro. A Practical Guide to the System Usability Scale: Background, Benchmarks \& Best Practices. Measuring Usability LLC, 2011.

[24] Heather L. O'Brien and Elaine G Toms. The Development and Evaluation of a Survey to Measure User Engagement. Journal of the American Society for Information Science and Technology, 61(1):50-69, 2010.

[25] Heather L. O'Brien and Elaine G Toms. Examining the Generalizability of the User Engagement Scale (UES) in Exploratory Search. Information Processing \& Management, 49(5):1092-1107, 2013.

[26] Jeff Sauro. Measuring usability with the system usability scale (SUS), 2011.

[27] Jacob Cohen. Statistical Power Analysis for the Behavioral Sciences. NJ: Lawrence Earlbaum Associates, 2, 1988.

[28] Jonathan Grudin. Partitioning Digital Worlds: Focal and Peripheral Awareness in Multiple Monitor Use. In Proceedings of the SIGCHI Conference on Human Factors in Computing Systems (CHI), pages 458-465. ACM, 2001. 